\title{
ANÁLISE DA ANSIEDADE TRAÇO COMPETITIVA EM JOVENS ATLETAS
}

\author{
Giovanna Carla Interdonato, Universidade Estadual de Londrina - UEL - Londrina, Paraná - Brasil \\ Arli Ramos de Oliveira, Universidade Estadual de Londrina - UEL - Londrina, Paraná - Brasil \\ Clovis Correa Luiz Júnior, Universidade Estadual de Londrina - UEL - Londrina, Paraná - Brasil \\ Márcia Greguol, Universidade Estadual de Londrina - UEL - Londrina, Paraná - Brasil
}

\section{RESUMO}

A ansiedade no contexto esportivo é um dos fatores psicológicos de muita relevância para os atletas, pois suas diferentes formas de manifestações poderão ou não influenciá-los no momento da competição. Assim, este estudo teve o objetivo de verificar os níveis de ansiedade-traço em atletas infanto-juvenis de diferentes modalidades esportivas. Para tanto foram analisados 73 atletas do sexo masculino, com média de idade de 13,2 anos $( \pm 1,88)$ e tempo de prática de 3,43 anos $( \pm 1,79)$, adeptos de basquetebol, futebol, judô, natação e voleibol. O instrumento de avaliação utilizado foi o "Sport CompetitionAnxiety Test (SCAT), desenvolvido por (MARTENS,1977), o qual avalia os níveis de ansiedade-traço competitiva. Os resultados demonstraram que os atletas, apesar de serem jovens, não apresentaram escores elevados de ansiedade-traço. As análises dos dados revelaram ainda que houve diferença estatisticamente significante nos níveis de ansiedade entre as modalidades individuais e coletivas.

Palavras-Chave: Ansiedade; Atletas; Jovens.

\section{ANALYSIS OF COMPETITIVE TRAIT ANXIETY IN YOUNG ATHLETES}

\begin{abstract}
The anxiety in the sportive context is one of the psychological issues of great relevance for the athletes, since its different forms of manifestation may or may not influence them at the moment of competition. So, this study had the objective to verify the levels of traceanxiety on young athletes of different sportive modalities. To do so were analyzed 73 athletes of male gender, with mean age of 13,2 years of old $( \pm 1,88)$ and activity time of 3,43 years $( \pm 1,79)$, basketball, soccer, judo, swimming and volleyball adepts. The evaluation instrument used was "Sport Competition Anxiety Test (SCAT), developed by (MARTENS,1977), which evaluates the levels of competitive trace-anxiety. The results showed that the athletes, despite their low age, did not present high trace-anxiety scores. The data analysis revealed too that there were significant statistical unbalance on the anxiety levels between the group and individual modalities.
\end{abstract}

KEY-WORDS: Anxiety; Athletes; Young. 


\section{INTRODUÇÃO}

O esporte é um meio no qual se vivencia as emoções com muita intensidade. As competições despertam sentimentos não só nos atletas como também nos espectadores. Os processos emocionais podem acompanhar de forma regular e apoiar a ação esportiva, mas também podem perturbá-la ou até impedi-la, alterando o estado de ansiedade. ${ }^{1}$

A ansiedade é uma resposta emocional aversiva ao estresse, que resulta de uma avaliação de ameaça e é caracterizada por sentimentos subjetivos de preocupação e apreensão relativamente à possibilidade de dano físico ou psicológico, muitas vezes acompanhados de aumento da ativação fisiológica. ${ }^{2}$ Independentemente do nível do atleta e idade, a ansiedade é um dos fatores psicológicos mais frequentes no esporte competitivo.

De acordo com $\mathrm{Cruz}^{3}$ a ansiedade, assim como outros fatores psicológicos, é determinante para o desempenho. SegundoWeinberg e Gould, ${ }^{4}$ uma das principais causas da ansiedade é justamente a importância do momento, ou seja, quanto maior a importância, maior a probabilidade dos atletas apresentarem elevado nível de ansiedade.

Para descrever e compreender melhor o fenômeno da ansiedade, alguns estudos ${ }^{5-7}$ consideraram a divisão da ansiedade em duas medidas: o traço de ansiedade, que representa uma característica, uma propensão para sentir maior ou menor grau de ansiedade diante de diferentes situações, e o estado de ansiedade, que mostra as reações do indivíduo perante situações temporárias ou tensões situacionais, observada em dado momento na vida do indivíduo.

De acordo com a distinção de ansiedade-estado e ansiedade-traço, segundo Spielberg, ${ }^{8}$ após um evento externo ou um estímulo interno ser percebido e avaliado como perigoso ou ameaçador o indivíduo:

Conexões: revista da Faculdade de Educação Física da UNICAMP, Campinas, v. 8, n. 3, p.1-9, set./dez. 2010. 
- evocará uma reação de estado de ansiedade que inclui ativação do Sistema Nervoso Autônomo e sentimentos subjetivos de tensão e expectativa;

- a intensidade desta reação será proporcional à quantidade de ameaça que a situação representa para o indivíduo;

- a ansiedade-estado permanecerá elevada até a avaliação da situação como ameaçadora ser alterada por estratégias de confronto ou comportamentos defensivos eficazes.

Spielberger $^{7}$ defende ainda que diferenças individuais na ansiedade-traço determinem os estímulos externos específicos que são cognitivamente avaliados como ameaçadores, o nível de ansiedade-estado experienciado e outros efeitos desses estímulos no comportamento.

A criança inserida no esporte deverá competir quando atingir seu estado de prontidão competitiva, que acontece a partir do equilíbrio de fatores como o crescimento, o desenvolvimento e a maturação sexual. Definir uma idade torna-se difícil devido às diferenças individuais. Entretanto, como referencial, acredita-se que isto deva ocorrer a partir dos 12 anos de forma genérica. ${ }^{9}$ Estudos que têm como tema principal a ansiedade esportiva comparada à faixa etária têm mostrado que os grupos mais jovens apresentam níveis de ansiedade maiores pelo fato de serem mais inexperientes. ${ }^{5}$

Tendo em vista a relevância do tema, este estudo tem como objetivo identificar os níveis de ansiedade traço competitiva em atletas infanto-juvenis de diferentes modalidades, uma vez que o conhecimento de tais níveis poderá contribuir para o rendimento destes jovens atletas durante a competição.

\section{METODOLOGIA}

A pesquisa caracterizou-se como transversal retro analítica, com base em dados primários. Teve como critério de inclusão sujeitos com idades entre 10 e 17 anos e tempo de prática esportiva maior que um ano.

Conexões: revista da Faculdade de Educação Física da UNICAMP, Campinas, v. 8, n. 3, p.1-9, set./dez. 2010. 
A amostra foi composta por 73 atletas, todos do sexo masculino, com idade média de 13,2 anos $( \pm 1,88)$ e tempo de prática de 3,43 anos $( \pm 1,79)$, adeptos de basquetebol $(n=14)$, futebol $(n=19)$, judô $(n=12)$, natação $(n=18)$ e voleibol $(n=10)$ em clubes e academias das cidades de Londrina/PR e de Botucatu/SP.

Como instrumento de avaliação foi utilizado o Sport CompetitionAnxiety Test (SCAT), desenvolvido por Martens ${ }^{10}$ e traduzido e adaptado por De Rose Junior eRosamilha citado por De Rose Junior. ${ }^{11}$ Este teste avalia os níveis de ansiedade-traço competitiva, e é composto por 15 perguntas que descrevem como o indivíduo se sente dentro da dada situação. A classificação é dada em escores, sendo oito itens de ativação, dois de desativação e cinco de efeito placebo. A pontuação é de um a três para os itens de ativação, e de três a um para os de desativação, sendo que o escore total varia de 10 (baixa ansiedade) a 30 (alta ansiedade). Antes da aplicação do instrumento de ansiedade, foi aplicado aos atletas um Termo de Consentimento Livre e Esclarecido, com a autorização do treinador, tornando-os conhecedores de todos os procedimentos da pesquisa realizada.

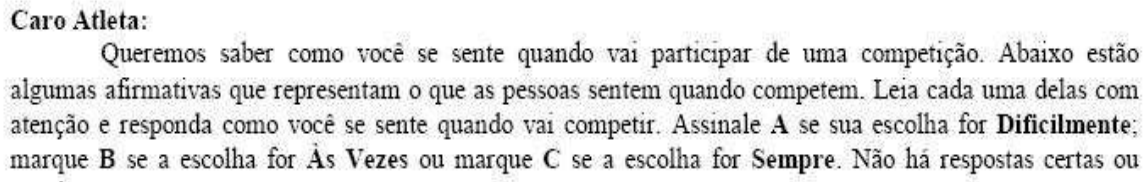

Queremos saber como você se sente quando vai participar de uma competição. Abaixo estão algumas a firmativas que representam o que as pessoas sentem quando competem. Leia cada uma delas com atenção e responda como você se sente quando vai competir. Assinale A se sua escolha for Dificilmente; marque B se a escolha for Às Vezes ou marque C se a escolha for Sempre. Não há respostas certas ou erradas.

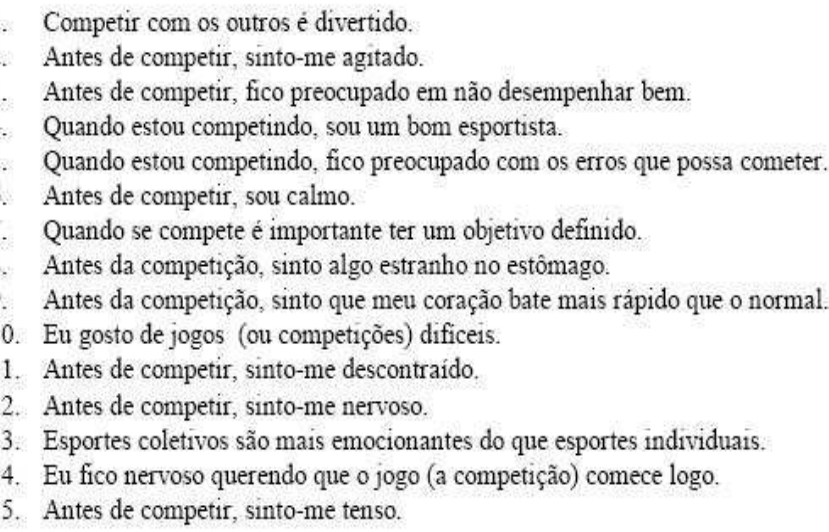

$\begin{array}{lll}\text { A } & \text { B } & \text { C } \\ \text { A } & \text { B } & \text { C } \\ \text { A } & \text { B } & \text { C } \\ \text { A } & \text { B } & \text { C } \\ \text { A } & \text { B } & \text { C } \\ \text { A } & \text { B } & \text { C } \\ \text { A } & \text { B } & \text { C } \\ \text { A } & \text { B } & \text { C } \\ \text { A } & \text { B } & \text { C } \\ \text { A } & \text { B } & \text { C } \\ \text { A } & \text { B } & \text { C } \\ \text { A } & \text { B } & \text { C } \\ \text { A } & \text { B } & \text { C } \\ \text { A } & \text { B } & \text { C } \\ \text { A } & \text { B } & \text { C }\end{array}$

Figura 1:Instrumento Sport Competition Anxiety Test (SCAT). Fonte: $^{11}$

A aplicação do Instrumento de Ansiedade Traço Competitiva foi realizada para cada atleta pertencente à amostra de forma anônima e foi preenchido voluntariamente. Considerandose que a variável estudada reflete um traço psicológico, no caso o fato do atleta ser ou não 
ansioso, a coleta de dados pode ser feita em qualquer momento do processo competitivo, segundo orientação do próprio autor do teste.

No plano analítico estatístico, foram realizadas análises descritivas, com médias e desviospadrão para verificar níveis de ansiedade das modalidades. A matriz de correlação linear (correlação de Pearson) foi utilizada para verificar as correlações entre os fatores idade e níveis de ansiedade e entre tempo de prática e níveis de ansiedade. Para analisar os níveis de ansiedade entre as modalidades foi realizada análise de variância (ANOVA) com teste post-hoc de Scheffé. Ao final, a amostra foi dividida em dois grupos - modalidades individuais e coletivas - a fim de se analisar as possíveis diferenças entre os níveis de ansiedade destes grupos, utilizando-se o test t de Student para amostras independentes Em todos os casos foram adotados níveis de significância $\mathrm{p} \leq 0,05$.

\section{RESULTADOS}

A tabela 1 a seguir mostra as médias e desvios padrões da idade, tempo de prática, níveis e os escores de ansiedade-traço dos atletas divididos por modalidades.

Tabela 1: Média dos valores das variáveis estudadas de acordo com cada modalidade

\begin{tabular}{lccccccl}
\hline Modalidades & Idade & D.P. & $\begin{array}{c}\text { Tempo } \\
\text { de } \\
\text { Prática }\end{array}$ & D.P. & $\begin{array}{c}\text { Níveis de } \\
\text { Ansiedade }\end{array}$ & D.P. & Escores \\
\hline Basquetebol & 13,57 & $\pm 1,5$ & 2,07 & $\pm 0,99$ & 21,14 & $\pm 3,08$ & Médio \\
Futebol & 14,52 & $\pm 1,26$ & 6 & $\pm 3,05$ & 21,10 & $\pm 3,98$ & Médio \\
Judô & 15,4 & $\pm 1,5$ & 3,08 & $\pm 1,16$ & 23,5 & $\pm 4,1$ & Médio-Alto \\
Natação & 10,8 & $\pm 1,23$ & 4,4 & $\pm 2,59$ & 23,2 & $\pm 3,89$ & Médio-Alto \\
Voleibol & 11,9 & $\pm 1,79$ & 1,6 & $\pm 0,69$ & 21,1 & $\pm 2,55$ & Médio \\
& & & & & & & \\
\hline TOTAL & 13,23 & $\pm 2,18$ & 3,78 & $\pm 2,66$ & 22,05 & $\pm 3,73$ & \\
\hline
\end{tabular}

Comparando-se as médias de cada modalidade, pode-se constatar que os atletas de modalidades individuais apresentaram níveis e consequentemente escores maiores de ansiedade-traço. No entanto, quando realizado o teste ANOVA para comparação de médias por modalidade uma a uma, verificaram-se valores sem significância estatística, como demonstrada na tabela 2 . 
Tabela 2: Análise de variância (ANOVA) por modalidade uma a uma.

\begin{tabular}{ccc}
\hline Modalidade & $\begin{array}{c}\text { Modalidades } \\
\text { Comparadas }\end{array}$ & Significância \\
\hline & Futebol & 1,00 \\
Basquetebol & Judô & 0,44 \\
& Natação & 0,48 \\
& Vôlei & 1,00 \\
Futebol & Basquetebol & 1,00 \\
& Judô & 0,36 \\
& Natação & 0,37 \\
& Vôlei & 1,00 \\
Judô & Basquetebol & 0,44 \\
& Futebol & 0,36 \\
& Natação & 0,99 \\
Natação & Vôlei & 0,51 \\
& Basquetebol & 0,48 \\
& Futebol & 0,37 \\
& Judô & 0,99 \\
Vôlei & Vôlei & 0,56 \\
& Basquetebol & 1,00 \\
& Futebol & 1,00 \\
& Judô & 0,51 \\
& Natação & 0,56 \\
\hline
\end{tabular}

Ao se realizar a matriz de correlação, não foram encontrados valores significativos nas correlações feitas entre a idade e os níveis de ansiedade $(\mathrm{p}=0,323)$ e entre tempo de prática e nível de ansiedade $(\mathrm{p}=0,372)$.

Quando verificada a influência do tempo de prática nos níveis de ansiedade, dividindo as amostras em 2 grupos, um com até 3 anos de prática e outro com mais de 3 anos de prática, novamente não foram encontradas diferenças significativas $(\mathrm{p}=0,589)$.

No entanto, quando comparada às médias dos níveis de ansiedade traço, através do teste $\mathrm{t}$ de Student, entre modalidades individuais e coletivas, foi constatada diferença significativa $(\mathrm{p}=0,009)$.

\section{DISCUSSÃO}

Os resultados encontrados neste estudo realizado com jovens atletas apresentam semelhanças com pesquisas realizadas por outros autores ${ }^{5,12-14}$ onde os achados mostraram 
que as médias dos níveis de ansiedade-traço apresentada por estes atletas de acordo com a escala estabelecida pelo autor do SCAT, ${ }^{10}$ no geral, não se apresentaram elevadas apesar da amostra ser constituída por jovens, indicando a possibilidade deles serem capazes de controlar sua ansiedade durante a prática esportiva apesar da pouca idade.

No entanto, quando foram verificados neste estudo os níveis de ansiedade traço competitiva entre modalidades individuais e coletivas através do teste $t$ (Student), houve diferenças estatisticamente significativas entre ambas. Outras pesquisas comprovam esta tendência mesmo sendo realizadas com outros tipos de modalidades. ${ }^{15,10}$ De acordo com os autores, em esportes individuais os atletas não compartilham a responsabilidade, expondose sozinhos a uma avaliação direta contribuindo assim para que pressões físicas e psíquicas tornem-se mais presentes, podendo levar ao aumento dos níveis de ansiedade. Além disso, especula-se também que nos esportes coletivos a presença dos companheiros de equipe talvez possa contribuir para a diminuição da responsabilidade individual diante dos resultados das competições, explicando a maior elevação da ansiedade dos atletas em esportes individuais.

Apesar da influência da idade nos níveis de ansiedade não ter sido significativa neste presente estudo, talvez pelo fato de o tempo de prática destes atletas ser considerável para suas respectivas idades, é importante ressaltar que os treinadores, os pais, e todos os outros profissionais envolvidos com o contexto esportivo devem respeitar a idade destes atletas, seus limites psicológicos e biológicos, pois estes podem influenciar na qualidade das experiências esportivas destes jovens, principalmente no desenvolvimento do seu autoconceito e do controle emocional, podendo afetar ou não o desenvolvimento destes atletas no esporte.

\section{CONCLUSÃO}

Pode-se concluir que os níveis de ansiedade - traço apresentados por estes jovens atletas de diferentes modalidades não se apresentaram elevados, mostrando significância apenas quando comparados entre modalidades coletivas e individuais. No entanto devemos destacar que ao analisar cada indivíduo deve-se levar em conta as diferenças individuais de cada um, incluindo características como personalidade, fatores sociais e estilo de vida. 
Partindo desta perspectiva, é fundamental o técnico saber como o seu atleta, lida e reage com a ansiedade, se ele a percebe como um fator positivo e facilitador ou negativo e prejudicial ao seu desempenho. Este estudo sugere ainda pesquisas complementares sobre a ansiedade-traço podendo esta ser realizada com outras modalidades.

\section{REFERÊNCIAS}

${ }^{1}$ COZZANI, M. Ansiedade no esporte. In: MACHADO, A. A. (Org.). Psicologia do esporte:temas emergentes. Jundiaí: Ápice, 1997, v. 1.

${ }^{2}$ SMITH, R. E.; SMOLL, F. L.; WIECHMAN, S. A. Measuring trait anxiety in sport. In: DUDA, J. L. (Ed.).Advances in sport and exercise psychology measurement Morgantown: fitness information technology, 1998. p. 105-127.

${ }^{3}$ CRUZ, J. F. A. Stress, ansiedade e competências psicológicas em atletas de elite e de alta competição: relação com o sucesso desportivo. In: ___ GOMES, A. R. (Ed.).Psicologia aplicada ao desporto e àactividade física. Braga: Univ. do Minho, 1997.

4 WEINBERG, R. S.; GOULD, D. Fundamentos da psicologia do esporte e do exercício. Porto Alegre: Artmed, 2001.

5 DE ROSE JUNIOR, D.; VASCONCELOS, E. G. Ansiedade-traço competitiva e atletismo: um estudo com atletas infanto-juvenis. Revista Paulista de Educação Física, São Paulo, v. 11, n. 2, p. 148-154, 1997.

${ }^{6} \mathrm{MACHADO}, \mathrm{A}$. A. Interferência da torcida na ansiedade e agressividade de atletas adolescentes. 1998. 186f. Tese do Instituto de Biociências, Universidade Estadual Paulista, Rio Claro.

${ }^{7}$ SPIELBERGER, C. D. Anxiety and behavior. New York: Academic Press, 1966.

8 SPIELBERGER, C. D. Stress and anxiety in sports. In: HACKFORT, D.; SPIELBERGER, C. D. (Ed.). Anxiety in sports: an international perspective. New York: Hemisphere, 1989. p. 3-17.

${ }^{9}$ MALINA, R. Competitive youth sports and biological maturation. In: BROWN, E.V.; BRANTA, C. F. (Ed.). Competitive sports for children and youth: an overview of research and issues. Champaign: Human Kinetics, 1988. p. 227-245.

${ }^{10}$ MARTENS, R. Social psychology and physical activity: problems in athletics New York: Harper and Row, 1977. p. 9-17.

${ }^{11}$ DE ROSE JUNIOR, D. Influência do grau de ansiedade-traço no aproveitamento de lances-livres. 1985. 59f. Dissertação (Mestrado EM Educação Física) - Escola de Educação Física, Universidade de São Paulo, São Paulo, 1985. 
${ }^{12}$ GONCALVES, M. P.;BELO, R. P. Ansiedade-traço competitiva: diferenças quanto ao gênero, faixa etária, experiência em competições e modalidade esportiva em jovens atletas. PsicoUSF, v.12, n.2, p.301-307, dez. 2007.

${ }^{13}$ INTERDONATO, G. C.; OLIVEIRA, A. R.; GORGATTI, M. G. Analise da ansiedadetraço em atletas infanto-juvenis de diferentes modalidades. In: SIMPÓSIO INTERNACIONAL DE CIÊNCIAS DO ESPORTE, 31.,2008, São Paulo. Anais...São Paulo: CELAFISCS, 2008.

${ }^{14}$ SANTOS, S. G.; PEREIRA, S. A. Perfil do nível de ansiedade-traço pré-competitiva de atletas de esportes coletivos e individuais do Estado do Paraná. Movimento, Porto Alegre, ano 3, n. 6, p. 3-12, 1997.

15 BECKER JÚNIOR, B. Manual de psicologia do esporte e exercício. Porto Alegre: Nova Prova, 2000. 